\title{
Comparison of Methods to Improve Fracture Risk Assessment in Chinese Diabetic Postmenopausal Women: A Case-Control Study
}

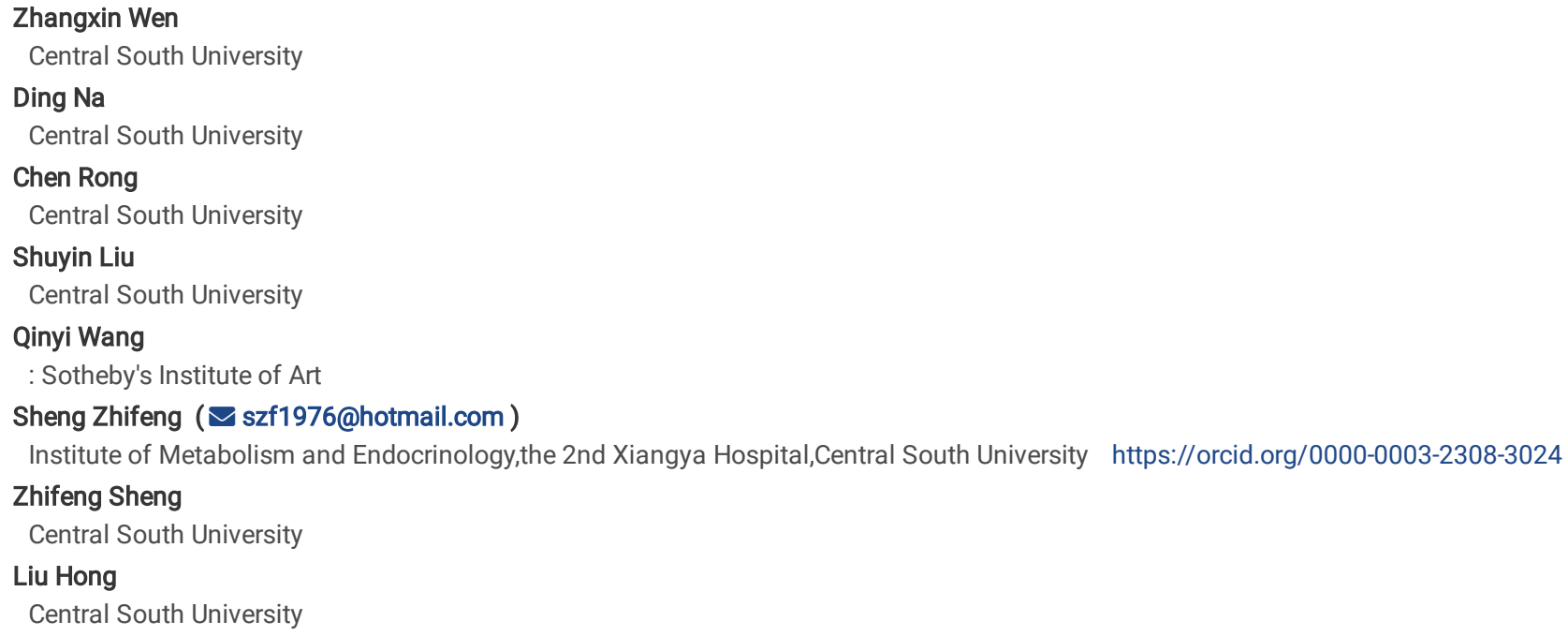




\section{Abstract}

Purpose

This study compared the performance of three proposed Fracture Risk Assessment Tool (FRAX) alternatives to the current standard Chinese FRAX in predicting bone fracture risk in type 2 diabetic (T2DM) postmenopausal women, and to explore the optimal strategy to better predicted fracture risk in postmenopausal women with diabetes in China.

Methods

We recruited 434 patients from community-medical centers, 217 with T2DM and 217 without T2DM (non-T2DM). All participants completed self-reported questionnaires detailing their characteristics and risk factors. Bone mineral density (BMD) and spinal radiographs were evaluated. The China FRAX model calculated all scores. The area under the receiver operator characteristic curve (ROC-AUC) evaluated the sensitivity, specificity, and accuracy for predicting 10year risk for major (MOF) and hip (OHF) osteoporotic fractures in T2DM patients.

Results

T2DM patients had higher BMD but lower average FRAX values than non-T2DM patients. The unadjusted FRAX ROC-AUC was 0.774, significantly smaller than that for 0.5 -unit femoral neck T-score-adjusted FRAX $(0.800 ; p=0.004)$. Rheumatoid arthritis (RA; $\mathrm{AUC}=0.810, p=0.033)$ and T-score $(\mathrm{AUC}=0.816, p=0.002)$ adjustments significantly improved fracture prediction in T2DM patients. Conclusions: Femoral neck T-score adjustment might be the preferred method for predicting MOF and OHF in Chinese diabetic postmenopausal women, while RA adjustment only effectively predicted HF risk.

Conclusions

Femoral neck T-score adjustment might be the preferred method for predicting MOF and OHF in Chinese diabetic postmenopausal women, while RA adjustment only effectively predicted HF risk.

\section{Introduction}

Osteoporosis is a systemic skeletal disease characterized by low bone mass and microarchitectural deterioration of bone tissue that leads to increased fracture risk [1], disability, financial burden, and mortality [2-5]. Therefore, it is essential to screen individuals at elevated risk of fractures in a timely manner.

The most widely used assessment instrument is the Fracture Risk Assessment Tool (FRAX $\left.{ }^{\circledR}\right)$. This computer-based algorithm predicts the 10-year probability of major osteoporotic fractures (MOF) (hip, clinical spine, forearm, and humerus fracture) and osteoporotic hip fractures (OHF) in the presence of competing mortality. The probability of fractures is based on the anamnestic, clinical factors, and anthropometric parameters, and considers, when available, the femoral neck bone mineral density (BMD) that can improve the accuracy of the fracture risk assessment [6].

The number of patients with osteoporosis in China is increasing with the aging population [7]. Menopause is a risk factor for diabetes and osteoporosis [8,9], and diabetes mellitus, particularly type 2 diabetes mellitus (T2DM), is a common disease in China. It was estimated in 2017 that the T2DM incidence in China was $10.4 \%$ [10]. T2DM is an independent risk factor for osteoporotic fractures, even though the femoral neck and lumbar BMD do not decrease in these patients [11-13]. The underlying mechanisms are not clear, but impaired bone strength and quality, falls, and bone strength alterations could explain this paradox [11,14]. T2DM confers an increased risk of fractures independently of the FRAX assessment with BMD [15]. However, the current FRAX formulation does not include T2DM among the risk factors. Moreover, evidence suggests that the FRAX algorithm does not reflect the risk of fractures in T2DM patients correctly [16].

There are several proposals on how to improve the FRAX performance for those with T2DM. Leslie et al [17,18] sought to improve FRAX predictive value by selecting rheumatism arthritis (RA) as an equivalent variable for T2DM among the fracture risk factors listed in the FRAX algorithm. Valentini et al. reported that a correction factor derived from hemoglobin A1c (HbA1c) could enhance the FRAX algorithm ability to predict the fracture risk for T2DM patients [19]. A observation reported that a T-score in a woman with DM is associated with hip fracture risk equivalent to a woman without DM with a T-score of approximately 0.5 units lower[15]. A recent study compared four proposed methods to improve FRAX performance for T2DM in male and female Canadians aged $>40$ years. The study suggested that each of the proposed methods could improve performance for individuals with diabetes. However, no method optimal in all settings was identified [20]. Therefore, the preferred approach is still unknown. Furthermore, no related studies assessed the adjusted FRAXs in a Chinese population and specifically in Chinese postmenopausal women with diabetes. Our recent study reported that the current recommended FRAX with BMD threshold guidelines have low sensitivity in the Chinese population [21]. Therefore, the primary aim of this study was to find which strategy (RA adjustment, T-score lowered by 0.5 of the standard deviation [SD], or adding ten years to the age) better predicted fracture risk in postmenopausal women with diabetes in China.

\section{Methods}

\section{Ethics statement}

The investigation has been conducted following the ethical standards of the Declaration of Helsinki and national and international guidelines. This study has been approved by the Ethics Committee of Xiangya Second Hospital, South China University, Changsha, China. All participants provided written informed consent. 


\section{Participants}

This is a case-control study. Postmenopausal women $(n=434)$ were recruited from community medical centers in Changsha City, Hunan Province, China, in September 2017. These included 217 T2DM patients and 217 consecutive postmenopausal women without diabetes as a control group. All participants had undergone baseline dual x-ray absorptiometry (DXA) of the lumbar spine, femoral neck and hip. The control and T2DM groups were matched for age and BMI. Menopause was defined as the absence of menstrual cycles for over one year. Exclusion criteria included the use of antiosteoporosis medication, presence of a malignant tumor, acute infection, and severe renal dysfunction. All participants completed standard medical assessment questionnaires to collect data about their medical, social, and family history, and clinical risk factors of FRAX. These factors included a parental history of hip fracture, history of fragility fractures, exposure to systemic glucocorticoids for more than three months, smoking, high alcohol intake (an average of three or more units daily), presence of RA, secondary osteoporosis factors, adult osteogenesis deficiency, long-term untreated hyperthyroidism, hypogonadism or premature menopause (<45 years), chronic malnutrition or malabsorption, and chronic liver disease.

\section{Assessment measures}

Height was measured to the nearest $0.5 \mathrm{~cm}$, using a stadiometer mounted on a wall and pressing a horizontal plate onto the head to flatten the hair. An electronic scale measured weight to the nearest $0.1 \mathrm{~kg}$. The BMI was calculated by dividing the body weight by the squared height $\left(\mathrm{kg} / \mathrm{m}^{2}\right)$. The participants age was recorded.

DXA scans were performed for the left femoral neck, total hip, and lumbar spine following the manufacturer's guidelines (Discovery Wi S/N87556, Hologic, USA), and they were measured by an experienced doctor. Osteoporosis was defined as a T-score $\leq-2.5$ following the World Health Organization recommendations used to establish the BMD reference database by our group [22]. Positive and lateral vertebral radiographs (T4 to L4) were acquired (UniVision 61Y040, Shimadzu, Japan) to assess vertebral fractures, using a visual semi-quantitative method. Fractures were defined as a ratio of the anterior to posterior or middle to posterior vertebral height $<0.8$, or the posterior vertebral height compared to that of an adjacent vertebra [23].

Base on the guidelines for diagnosis and treatment of primary osteoporosis in China, osteoporosis was diagnosed if a patient met any of the following criteria: 1) fragility fractures of the hip or vertebra; 2) T-score of less than -2.5 for BMD measured by DXA in the medial axial skeleton; 3 ) low bone mass based on the BMD results $(-2.5<$ T-score $<-1.0)$ with fragile fractures of the proximal humerus, pelvis, or distal forearm [24]. Diabetes diagnosis followed the guidelines for the prevention and control of T2DM in China (2017 Edition) [10].

The 10-year risk for MOF, including OHF, were calculated using the China FRAX ${ }^{\circledR}$ model, with the femoral neck T-score included in the calculations. We compared three alternative options to enhance the performance of FRAX in patients with diabetes. First, FRAX with RA input as a proxy for the effect of diabetes; this is justified by the similar weights assigned to RA and T2DM in the QFracture algorithm [25]. Second, FRAX with the femoral neck T-score input lowered by 0.5 SD in T2DM patients; other studies have observed that women with and without T2DM and equivalent OHF risk differed in their T-score by approximately 0.5 SD [35]. Third, FRAX with a 10-year higher age input for T2DM patients. This age correction is equivalent to an expected femoral neck BMD loss of $0.5 \mathrm{SD}$ over ten years.

\section{Statistical analysis}

Continuous variables are presented as mean \pm SD. Normal distribution of continuous variables was assessed separately for diabetic and nondiabetic participants by the Kolmogorov-Smirnov test. Categorical variables were analyzed by the Chi-squared test. We constructed ROC curves to determine the AUC and the corresponding $95 \%$ confidence intervals to identify the reference variable. The AUC is an overall estimate of the risk score accuracy in identifying the optimal choice [26]. The AUCs were compared using Stata 14.0 (Stata Corp, College Station, Tx). The cutoff value for statistical significance was set at $p<$ 0.05. The best cutoff point in the AUCs was estimated using the Youden index, and the accuracy of the thresholds was assessed by analyzing their sensitivity, specificity, and likelihood ratios. All data management and part of the analysis were performed using SPSS Statistics for Windows, Version 24.0 (SPSS Inc., Chicago, IL, USA).

\section{Results}

Characteristics of the 434 postmenopausal participants are summarized in Table 1. The average ages were $64.5 \pm 7.3$ and $65.0 \pm 8.4$ years in the T2DM and control groups, respectively. The groups were similar for menopause age, BMI, current smoking, drinking, and secondary osteoporosis. The mean unadjusted FRAX values for the probability of MOF and OHF in the T2DM group were higher than in the control group ( $3.69 \pm 1.85$ vs. $5.12 \pm 3.29$ and $0.93 \pm 1.07$ vs. 1.82 \pm 2.32 , respectively, $p<0.001$ for both). Mean lumber, hip, and especially femoral neck BMD values were higher in the T2DM group, but the differences did not reach the statistical significance level. Although T2DM patients had a higher BMD and a lower FRAX score, according to China's guidelines for the diagnosis and treatment of primary osteoporosis, fragility fractures occurred in 53 patients (24.4\%) in the T2DM group and only 14 (6.5\%) in the control group. Osteoporosis occurred in 94 (43.4\%) and 68 (31.3\%) patients in the T2DM and control groups, respectively (Table 2). The areas under the curves (AUCs) were compared between the T2DM and control groups for the original MOF FRAX and OHF FRAX (Table 3). The AUCs for MOF and OHF FRAX in the control group were significantly larger than in the T2DM group ( 0.94 vs. 0.77 and 0.93 vs. 0.79 , respectively, $p<0.001$ for both). All adjusted FRAX methods showed significantly higher values for OHF and MOF than the unadjusted FRAX (Figure 1a, b).

The AUC for predicting the 10-year risk of MOF was 0.774 for the unadjusted algorithm, 0.786 after RA adjustment, 0.790 after age adjustment, and 0.800 after T-score adjustment (Table 4). Similarly, the AUC for predicting the 10-year risk of OHF was 0.787 for unadjusted FRAX, 0.810 after RA adjustment, 0.811 after age adjustment, and 0.816 after T-score adjustment (Table 5). Lowering the femoral neck T-score by 0.5 SD, raising the age by ten years, and RA adjustment could all improve the performance of MOF and OHF FRAX. However, the only significant difference between the unadjusted and adjusted MOF 
FRAX was observed after lowering the femoral neck T-score by 0.5 SD ( $p=0.0041$; Table 4). The receiver operator characteristic (ROC) analysis showed that RA $(p=0.033)$ and T-score $(p=0.002)$ adjustments could significantly improve osteoporotic OHF prediction in diabetic women (Table 5). There was no difference between the RA and T-score adjusted FRAXs $(p=0.48)$.

The sensitivity and specificity of the 10-year unadjusted MOF probability and T-score adjusted FRAX for detecting osteoporosis were 0.683 and 0.817 , and 0.743 and 0.673 , respectively. The accuracy of BMD T-score adjusted FRAX was higher than the unadjusted FRAX (Table 4). The sensitivity and specificity of the unadjusted OHF probability and T-score adjusted FRAX for detecting osteoporosis were 0.750 and 0.798 , and 0.708 and 0.699 , respectively. The positive likelihood ratios and accuracy of T-score and RA adjusted FRAXs were higher, and negative likelihood ratios were lower than in the unadjusted FRAX (Table 5).

\section{Discussion}

A large body of evidence has suggested that diabetes increases fracture risk despite the presence of normal or high BMD [12,27,28]. Bonaccorsi et al. [24-29] performed a study in postmenopausal Italian women with and without diabetes. They found no difference between the groups in terms of BMD. Our study also demonstrated this perplexity. There were also no differences in age and BMI between the two groups (T2DM and controls). This homogeneity may explain why our T2DM patients presented with similar BMD. An increased occurrence of fragility fracture was reported in patients with T2DM, especially in Asians [30]. We also detected a high prevalence of fractures in the T2DM group. The BMD had relatively low sensitivity for detecting high fracture risk in diabetes patients, as indicated by results showing that some fragile fractures occur when T-score $>-2.5$. While this value cannot diagnose osteoporosis, the phenomenon is apparent in diabetes patients. Previous studies have shown, and we confirmed, that BMD does not discriminate against T2DM patients with a higher risk of osteoporotic fractures [31].

The FRAX was developed to remedy the limitation of BMD in predicting fractures. Therefore, predictive tools like FRAX are recommended for identifying individuals at a higher risk of osteoporotic fractures in many clinical guidelines [32,33]. Our study showed that the mean FRAX score values were significantly lower in patients with T2DM, but the prevalence of osteoporosis and fragility fractures in these patients was much higher. These findings concur with those of other studies that showed T2DM subjects to have a higher incidence of fractures than the controls [13] and that FRAX underestimates the fracture risk in diabetes patients [34,35]. This bias is evident in postmenopausal women [36]. The femoral neck BMD is a dominant factor in the FRAX risk assessment. We found both study groups to have similar femoral neck BMD. Therefore, if the reference standard of osteoporosis is based on BMD measurement at the femoral neck, it will miss most patients. The emerging conclusion is that the current FRAX algorithm might be inappropriate for T2DM patients, and further studies are required to optimize methods to identify postmenopausal women with T2DM at an elevated risk of fractures.

Many methods have been put forth to address the problem of fracture risk assessment in postmenopausal women with T2DM. These include making a Trabecular Bone Score (TBS) adjustment to FRAX, increasing the age by ten years, decreasing the femoral neck BMD T-score by 0.5 SD, or using "Diabetes" to represent "Rheumatoid Arthritis" in FRAX. However, these alternatives were not validated in different settings, including Chinese postmenopausal women with T2DM. To the best of our knowledge, this is the first study to directly compare the performance of the proposed adjusted FRAX methods to improve the fracture risk prediction among individuals with T2DM in the Chinese population. All adjustments led to an improvement in FRAX performance by reducing or eliminating the effect of diabetes on the incidence of MOF and OHF. The 10-year probability of OHF was more accurate than the 10-year probability of a MOF, which is consistent with the results of other studies [37]. Many have suggested that simultaneous estimation of fracture risk in many sites could increase the risk assessment performance because risk factors for fractures, or the magnitude of their effect, differ across fracture sites [38-41].

We observed that FRAX with a lowered T-score had a significantly higher MOF discriminative ability than unadjusted FRAX. Lowering the T-score and substituting T2DM for RA significantly improved FRAX assessment of OHF risk, but BMD sensitivity after altering the T-score was greater than after RA adjustment. With similar specificity between them, lowering the T-score can screen out more people with osteoporosis. Furthermore, RA adjustment can only be applied when RA and diabetes do not coexist in the same individual. This study showed that reducing the femoral neck T-score input to FRAX by 0.5 SD significantly improved the predictive ability of MOF and OHF risk for postmenopausal diabetes patients. These results suggest the T-score-adjusted FRAX algorithm to be potentially useful, at least for T2DM patients. Still, the Chines Experts Consensus recommended that when the FRAX tool is used in patients with diabetes, "Diabetes" should be used instead of "Rheumatoid Arthritis" in the tool [42]. This might be because data on age or BMD T-score differences between diabetic and non-diabetic patients with similar hip fracture risk in the Chinese populations are scarce. Our study found that changing the BMD T-score can eliminate the effect of diabetes in fracture assessment, at least in postmenopausal women.

In contrast to the study by Leslie et al. [20], increasing the age input to FRAX by ten years was similar to unadjusted FRAX in predicting the risk for MOF and OHF in our T2DM postmenopausal patients. This difference might be related to the homogeneity of our population in terms of sex and ethnicity. Besides, the average age of our diabetic women was 63.73 years, and $51 \%$ were older than 65 years, an age of increased fracture risk. We observed that using the FRAX score after substituting diabetes for RA performed better only in predicting OHF. Many studies verified that diabetes modifies the effectiveness of FRAX, particularly at the hip and other peripheral skeletal sites [40,43], and showed through 6-8 years of follow-up that the HF risk in T2DM patients was higher than in non-diabetes subjects [44]. The data reported here might help inform future position statements and practice guidelines aimed to enhance the care of diabetic postmenopausal women.

Our research has some limitations. First, the participants sample size was relatively small. The proposed adjusted methods require further testing in a larger population. Second, self-administered questionnaires might lead to recall and reporting biases. Third, there was no follow-up period, and future fractures were, therefore, missed. Our results need to be verified by prospective studies. Fourth, we did not include TBS-adjusted FRAX in this research because it is not widely used in China. Therefore, we could not compare the effectivity between BMD T-score adjusted and TBS-adjusted FRAX.

In Conclusion, FRAX underestimates the risk of osteoporosis-related fracture in postmenopausal women with diabetes. This underestimation delays osteoporosis treatment and interventions in these patients. Adjusted FRAX could significantly compensate for the effect of diabetes in Chinese diabetic 
postmenopausal women. Adjusted FRAX alternatives could serve as non-invasive methods for reliable bone quality assessment in patients with diabetes..

\section{Declarations}

Funding: This work was supported by grants from the National Nature Science Foundation of China (grant numbers 81471091 and 81870622 ) $\nabla$ the Hunan Nature Science Foundation (grant number 2018JJ2574) and Bethune Charitable Foundation『grant numbers G-X-2019-1107-3囚

Conflicts of interest: The authors declare that they have no conflict of interest.

Availability of data and material: data transparency.

Code availability: SPSS $₫$ Stata.

Authors' contributions: The study conception and design were performed by Sheng Zhifeng and Liu Hong. Data collection and analysis were performed by Wen Zhangxin, Ding Na, Chen Rong, Wang Qinyi. Data verification and the first draft of the manuscript was written by Wen Zhangxin, Ding Na. liu shuyin, Wang Qinyi and Chen Rong were responsible for statistical analysis. All authors approved the final manuscript and taking final responsibility for the paper.

Ethics approval: All research procedures in this study were in accordance with the ethical standards of institutions and with the 1964 Helsinki declaration and its subsequent amendments.

Consent to participate: Informed consent was obtained from all individual participants included in the study.

Consent for publication: Not applicable

\section{Acknowledgments:}

None

\section{References}

1. Consensus development conference: diagnosis, prophylaxis, and treatment of osteoporosis. J. Med. https://doi.org/10.1016/0002-9343(93)90218-e.

2. Center JR, Nguyen TV, Schneider D, Sambrook PN, and Eisman JA. Mortality after all major types of osteoporotic fracture in men and women: an observational study. Lancet(1999).https://doi.org/10.1016/s0140-6736(98)09075-8.

3. loannidis G, Papaioannou A, Hopman WM, Akhtar-Danesh N, Anastassiades T, Pickard L, Kennedy CC, Prior JC, Olszynski WP, Davison KS, Goltzman D, Thabane L, Gafni A, et al. Relation between fractures and mortality: results from the Canadian Multicentre Osteoporosis Study. CMAJ(2009). https://doi.org/10.1503/cmaj.081720.

4. Si L, Winzenberg TM, Jiang Q, Chen M, and Palmer AJ. Projection of osteoporosis-related fractures and costs in China: 2010-2050. Int (2015) https://doi.org/10.1007/s00198-015-3093-2.

5. Magnusson KA, Gunnarsson B, Sigurosson GH, Mogensen B, Olafsson Y, and Karason S Treatment and outcome of patients with hip fracture. Laeknabladid. (2016) https://doi.org/10.17992/lbl.2016.03.69.

6. Kanis JA, Hans D, Cooper C, Baim S, Bilezikian JP, Binkley N, Cauley JA, Compston JE, Dawson-Hughes B, El-Hajj Fuleihan G, Johansson H, Leslie WD, Lewiecki EM, et al Interpretation and use of FRAX in clinical practice. Osteoporos Int(2011) https://doi.org/10.1007/s00198-011-1713-z.

7. Cosman F, deBeur SJ, LeBoff MS, Lewiecki EM, Tanner B, Randall S, Lindsay R .National Osteoporosis Foundation, Clinician's Guide to Prevention and Treatment of Osteoporosis. Osteoporos Int(2015) https://doi.org/10.1007/s00198-014-2794-2.

8. Rathmann W, Giani G. Global prevalence of diabetes: estimates for the year 2000 and projections for 2030. Diabetes Care (2004) https://doi.org/10.2337/diacare.27.10.2568.

9. Brand JS, van der Schouw YT, Onland-Moret NC, Sharp SJ, Ong KK, Khaw KT, Ardanaz E, Amiano P, Boeing H, Chirlaque MD, ClavelChapelon F, Crowe FL, de Lauzon-Guillain B, et al. Age at menopause, reproductive life span, and type 2 diabetes risk: results from the EPIC-InterAct study. Diabetes Care (2013) https://doi.org/10.2337/dc12-1020.

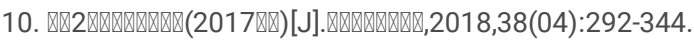

11. Oei L, Zillikens MC, Dehghan A, Buitendijk GH, Castaño-Betancourt MC, Estrada K, Stolk L, Oei EH, van Meurs JB, Janssen JA, Hofman A, van Leeuwen JP, Witteman JC, et, al.High bone mineral density and fracture risk in type 2 diabetes as skeletal complications of inadequate glucose control: the Rotterdam Study. Diabetes Care (2013) https://doi.org/10.2337/dc12-1188.

12. de Liefde II, van der Klift M, de Laet CE, van Daele PL, Hofman A, Pols HA. Bone mineral density and fracture risk in type-2 diabetes mellitus: the Rotterdam Study. Osteoporos Int (2005). https://doi.org/10.1007/s00198-005-1909-1.

13. Yamamoto M, Yamaguchi T, Yamauchi M, Kaji H, Sugimoto T. Diabetic patients have an increased risk of vertebral fractures independent of BMD or diabetic complications. J Bone Miner Res (2009). https://doi.org/10.1359/jbmr.081207.

14. Gregg EW, Beckles GL, Williamson DF, Leveille SG, Langlois JA, Engelgau MM, Narayan KM.Diabetes and physical disability among older U.S. adults. Diabetes Care(2000). https://doi.org/10.2337/diacare.23.9.1272.

15. Schwartz AV, Vittinghoff E, Bauer DC, Hillier TA, Strotmeyer ES, Ensrud KE, Donaldson MG, Cauley JA, Harris TB, Koster A, Womack CR, Palermo L, Black DA al. Association of BMD and FRAX score with risk of fracture in older adults with type 2 diabetes. JAMA (2011). https://doi.org/10.1001/jama.2011.715. 
16. Giangregorio LM, Leslie WD, Lix LM, Johansson H, Oden A, McCloskey E, Kanis JA. FRAX underestimates fracture risk in patients with diabetes. J Bone Miner Res (2012). https://doi.org/10.1002/jbmr.556.

17. Schacter GI, Leslie WD . DXA-Based Measurements in Diabetes: Can They Predict Fracture Risk? Calcif Tissue Int (2017). https://doi.org/10.1007/s00223016-0191-x.

18. Biver E. FRACTURE RISK ASSESSMENT IN DIABETES. Osteoporosis International (2018)

19. Valentini A, Cianfarani MA, De Meo L, Morabito P, Romanello D, Tarantino U, Federici M, Bertoli A. FRAX tool in type 2 diabetic subjects: the use of HbA $A_{1 c}$ in estimating fracture risk. Acta Diabetol(2018). https://doi.org/10.1007/s00592-018-1187-y.

20. Leslie WD, Johansson H, McCloskey EV, Harvey NC, Kanis JA, Hans D. Comparison of Methods for Improving Fracture Risk Assessment in Diabetes: The Manitoba BMD Registry. J Bone Miner Res (2018). https://doi.org/10.1002/jbmr.3538.

21. Liu S, Chen R, Ding N, Wang Q, Huang M, Liu H, Xie Z, Ou Y, Sheng Z.Setting the new FRAX reference threshold without bone mineral density in Chinese postmenopausal women. J Endocrinol Invest (2020). https://doi.org/10.1007/s40618-020-01315-4.

22. Sheng Z, Xu K, Ou Y, Dai R, Luo X, Liu S, Su X, Wu X, Xie H, Yuan L, Liao E.Relationship of body composition with prevalence of osteoporosis in central south Chinese postmenopausal women. Clin Endocrinol (Oxf) (2011). 74 :319-324. https://doi.org/10.1111/j.1365-2265.2010.03941.X.

23. Genant HK, Wu CY, van Kuijk C, Nevitt MC. Vertebral fracture assessment using a semiquantitative technique. J Bone Miner Res (1993). https://doi.org/10.1002/jbmr.5650080915.

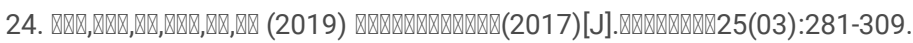

25. Hippisley-Cox J, Coupland C. Derivation and validation of updated QFracture algorithm to predict risk of osteoporotic fracture in primary care in the United Kingdom: prospective open cohort study. BMJ (2012). https://doi.org/10.1136/bmj.e3427.

26. Hanley JA, McNeil BJ. The meaning and use of the area under a receiver operating characteristic (ROC) curve. Radiology(1982). https://doi.org/10.1148/radiology.143.1.7063747.

27. Bonds DE, Larson JC, Schwartz AV, Strotmeyer ES, Robbins J, Rodriguez BL, Johnson KC, Margolis KL Risk of fracture in women with type 2 diabetes: the Women's Health Initiative Observational Study. J Clin Endocrinol Metab(2006). https://doi.org/10.1210/jc.2006-0614.

28. Kasperk C, Georgescu C, Nawroth P. Diabetes Mellitus and Bone Metabolism. Exp Clin Endocrinol Diabetes (2017). https://doi.org/10.1055/s-0042123036.

29. Bonaccorsi G, Fila E, Messina C, Maietti E, Ulivieri FM, Caudarella R, Greco P, Guglielmi G. Comparison of trabecular bone score and hip structural analysis with FRAX in postmenopausal women with type 2 diabetes mellitus. Aging Clin Exp Res(2017). https://doi.org/10.1007/s40520-016-0634-2.

30. Yamamoto M, Yamaguchi T, Yamauchi M, Kaji H, Sugimoto T. Diabetic patients have an increased risk of vertebral fractures independent of BMD or diabetic complications. J Bone Miner Res (2009). https://doi.org/10.1359/jbmr.081207.

31. Clark P, Denova Gutiérrez E, Zerbini C, Sanchez A, Messina O, Jaller JJ, Campusano C, Orces CH, Riera G, Johansson H, Kanis JA. FRAX-based intervention and assessment thresholds in seven Latin American countries. Osteoporos Int (2018). https://doi.org/10.1007/s00198-017-4341-4.

32. Nakamura T.Recommendations of FRAX in clinical assessment of osteoporosis indicated in European and US guidelines. Clin Calcium (2009). https://doi.org/CliCa091217231728.

33. Compston J, Cooper A, Cooper C, Gittoes N, Gregson C, Harvey N, Hope S, Kanis JA, McCloskey EV, Poole KES, Reid DM, Selby P, Thompson F et al.UK clinical guideline for the prevention and treatment of osteoporosis. Arch Osteoporos (2017). https://doi.org/10.1007/s11657-017-0324-5.

34. Leslie WD, Rubin MR, Schwartz AV, Kanis JA. Type 2 diabetes and bone. J Bone Miner Res (2012). https://doi.org/10.1002/jbmr.1759.

35. Schwartz AV, Vittinghoff E, Bauer DC, Hillier TA, Strotmeyer ES, Ensrud KE, Donaldson MG, Cauley JA, Harris TB, Koster A, Womack CR, Palermo L, Black DA et al. Association of BMD and FRAX score with risk of fracture in older adults with type 2 diabetes. JAMA (2011). https://doi.org/10.1001/jama.2011.715.

36. Majumdar SR, Leslie WD, Lix LM, Morin SN, Johansson H, Oden A, McCloskey EV, Kanis JA. Longer Duration of Diabetes Strongly Impacts Fracture Risk Assessment: The Manitoba BMD Cohort. J Clin Endocrinol Metab(2014). https://doi.org/10.1210/jc.2016-2569.

37. Chen XF, Li XL, Zhang H, Liu GJ. Were you identified to be at high fracture risk by FRAX® before your osteoporotic fracture occurred? Clin Rheumatol (2014). https://doi.org/10.1007/s10067-014-2533-2.

38. Johansson H, Kanis JA, Odén A, McCloskey E, Chapurlat RD, Christiansen C, Cummings SR, Diez-Perez A, Eisman JA, Fujiwara S, Glüer CC, Goltzman D, Hans D, et al. A meta-analysis of the association of fracture risk and body mass index in women. J Bone Miner Res (2014). https://doi.org/10.1002/jbmr.2017.

39. Beaudoin C, Jean S, Moore L, Gamache P, Bessette L, Ste-Marie LG, Brown JP. Number, Location, and Time Since Prior Fracture as Predictors of Future Fracture in the Elderly from the General Population. J Bone Miner Res (2018). https://doi.org/10.1002/jbmr.3526.

40. Janghorbani M, Van Dam RM, Willett WC, Hu FB. Systematic review of type 1 and type 2 diabetes mellitus and risk of fracture. Am J Epidemiol (2007). https://doi.org/10.1093/aje/kwm106.

41. Janghorbani M, Feskanich D, Willett WC, Hu F. Prospective study of diabetes and risk of hip fracture: the Nurses' Health Study. Diabetes Care (2006). https://doi.org/10.2337/dc06-0440.

42. Liu JM, Zhu DL, Mu YM, Xia WB. Management of fracture risk in patients with diabetes-Chinese Expert Consensus. J Diabetes (2019). https://doi.org/10.1111/1753-0407.12962.

43. Vestergaard P. Discrepancies in bone mineral density and fracture risk in patients with type 1 and type 2 diabetes-a meta-analysis. Osteoporos Int (2007). https://doi.org/10.1007/s00198-006-0253-4. 
44. Tebé C, Martinez-Laguna D, Moreno V, Cooper C, Diez-Perez A, Collins GS, Prieto-Alhambra D. Differential Mortality and the Excess Rates of Hip Fracture Associated With Type 2 Diabetes: Accounting for Competing Risks in Fracture Prediction Matters. J Bone Miner Res (2018).

https://doi.org/10.1002/jbmr.3435.

\section{Tables}

Table 1 Study population baseline characteristics

\begin{tabular}{|llll|}
\hline Characteristics & T2DM & Non-T2DM & P value \\
\hline Number & 217 & 217 & \\
Age, years & $64.5 \pm 7.28$ & $65.0 \pm 8.35$ & 0.623 \\
Age at menopause, year & $49.5 \pm 3.27$ & $49.7 \pm 3.72$ & 0.584 \\
\hline BMI, kg/m ${ }^{2}$ & $23.3 \pm 3.36$ & $23.4 \pm 3.07$ & 0.709 \\
\hline Prior fracture & $16 / 217$ & $13 / 217$ & 0.564 \\
\hline Smoke & $3 / 217$ & $4 / 217$ & 0.713 \\
Alcohol intake & $1 / 217$ & $4 / 217$ & 0.685 \\
Menopause at earlier age & $4 / 217$ & $16 / 217$ & 0.267 \\
Secondary osteoporosis & $21 / 217$ & $24 / 217$ & 0.637 \\
Lumbar spine BMD & $0.838 \pm 0.151$ & $0.814 \pm 0.140$ & 0.093 \\
Femur neck BMD & $0.646 \pm 0.166$ & $0.619 \pm 0.103$ & 0.298 \\
Hip BMD & $0.767 \pm 0.137$ & $0.766 \pm 0.119$ & 0.942 \\
\hline FRAX 10-year MOF fracture risk $\% \bigotimes$ & $3.69 \pm 1.85$ & $5.12 \pm 3.29$ & $<0.001$ \\
\hline FRAX 10-year HF fracture risk(\%) & $0.93 \pm 1.07$ & $1.82 \pm 2.32$ & $<0.001$ \\
\hline
\end{tabular}

T2DM : Type 2 diabetes ,MOF:major osteoporotic fracture, HF:hip fracture, RA: rheumatoid arthritis, BMI: body mass index, BMD:bone mineral density.

Table2 Diagnosis of osteoporosis based on diagnostic criteria

\begin{tabular}{|c|c|c|}
\hline Osteoporosis diagnosis criteria & T2DM & Non-T2DM \\
\hline BMD(lumber) $\leq-2.5$ & 72 & 44 \\
\hline BMD (lumber or femoral neck \hip) $\leq-2.5$ & 74 & 61 \\
\hline BMD (femoral neck) $\leq-2.5$ & 24 & 51 \\
\hline BMD(hip) $\leq-2.5$ & 56 & 17 \\
\hline Low bone mass with fragility fracture & 20 & 7 \\
\hline Fragility fracture $\otimes \% \bigotimes$ & $53 \llbracket 24.4 \rrbracket$ & $14 \otimes 6.5 \bigotimes$ \\
\hline Any criteria $₫ \% \bigotimes$ & $94 \bowtie 43.3 \rrbracket$ & $68 \rrbracket 31.3 \rrbracket$ \\
\hline
\end{tabular}

BMD:bone mineral density

Table 3 Area Under the Curve for Major Osteoporotic and Hip Fracture Discrimination in Individuals Without and With Diabetes

\begin{tabular}{|llllll|}
\hline & Non-T2DM & \multicolumn{3}{c|}{ T2DM } \\
\hline & AUC & $95 \% \mathrm{Cl}$ & AUC & $95 \% \mathrm{Cl}$ & P value \\
\hline Prediction of MOF from FRAX with BMD & 0.94 & $0.90-0.97$ & 0.77 & $0.71-0.84$ & $<0.001$ \\
\hline Prediction of HF from FRAX with BMD & 0.93 & $0.90-0.97$ & 0.79 & $0.73-0.85$ & $<0.001$ \\
\hline
\end{tabular}

MOF:major osteoporotic fracture, HF:hip fracture, AUC: area under the curve, Cl: confidence interval, BMD: bone mineral density

Table4 The comparison of the area of ROC between unadjusted ((referent) FRAX for major osteoporotic fracture and three adjustments. 


\begin{tabular}{|llllllll|}
\hline & Sensitivity & Specificity & Accuracy & Positive likelihood ratio & Negative likelihood ratio & AUC & P value \\
\hline FRAX MOF unadjusted & 0.683 & 0.743 & 0.714 & 2.66 & 0.427 & 0.774 & 0.272 \\
\hline With age raised 10 years & 0.827 & 0.637 & 0.728 & 2.28 & 0.293 & 0.790 & 0.252 \\
\hline With RA adjusted & 0.808 & 0.655 & 0.728 & 2.34 & 0.272 & 0.282 \\
\hline With T-score lowered 0.5 & 0.817 & 0.673 & 0.742 & 2.50 & 0.800 & 0.004 \\
\hline
\end{tabular}

FRAX: Fracture Risk Assessment, MOF:major osteoporotic fracture, RA: rheumatoid arthritis, AUC: area under the curve ,SD:standard deviation

Table5 The comparison of the area of ROC between unadjusted FRAX for hip osteoporotic fracture ((referent) and three adjustments.

\begin{tabular}{|llllllll|}
\hline & Sensitivity & Specificity & Accuracy & Positive likelihood ratio & Negative likelihood ratio & AUC & P value \\
\hline FRAX HF unadjusted & 0.750 & 0.708 & 0.728 & 2.57 & 0.353 & 0.787 & 0.252 \\
\hline With age raised 10 years & 0.837 & 0.646 & 0.737 & 2.36 & 0.296 & 0.811 & 0.101 \\
\hline With RA adjusted & 0.788 & 0.717 & 0.751 & 2.78 & 0.204 & 0.810 & 0.033 \\
\hline With T-score lowered 0.5 & 0.798 & 0.699 & 0.747 & 2.65 & 0.816 & 0.002 \\
\hline
\end{tabular}

FRAX: Fracture Risk Assessment, HF:hip fracture ,RA: rheumatoid arthritis, AUC: area under the curve, SD:standard deviation

\section{Figures}
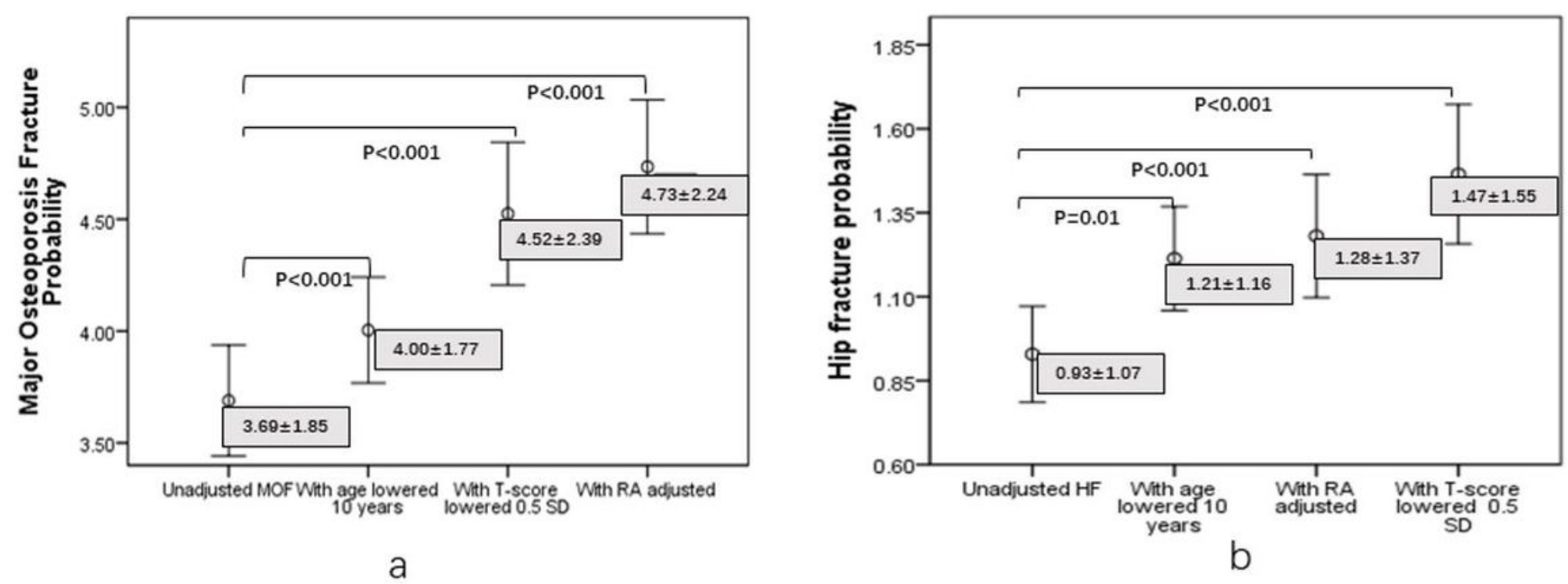

Figure 1

a Comparison of the average value of unadjusted major osteoporotic fracture and after three adjustments for major osteoporotic fracture applied to those with diabetes, b Comparison of the average value of unadjusted Hip fracture and after three adjustment for Hip fracture applied to those with diabetes. 